\title{
The high price of progress
}

The field of clinical oncology has never been more interesting than it is now. The past few years have seen notable improvements in the ability of oncologists to treat patients, even those who previously had a very dismal hope of survival. The opportunities provided by immunotherapies, the availablity of better targeted therapies, and more-sophisticated companion diagnostics have ushered an era of excitement and possibilities; however, in our data-laden landscape, an increased appreciation of the complexity of the multitude of diseases termed cancer, and our suboptimal trial approaches that do not always reflect the latest diagnostic and treatment advances or their application to real-world scenarios, have created great challenges.

As outlined in several newly-introduced 'Comment' articles published in this issue by experts in drug approval, regulation and affordability, oncologists and patients are increasingly having to decide about treatment plans by balancing the patient's clinical needs with their personal financial situation, or that of their national health-care system. Several measures have been implemented in an attempt to address this issue. The ASCO Value Framework (ASCO-VF) and ESMO Magnitude of Clinical Benefit Scale (ESMO-MCBS) have been introduced with the aim of quantifying the benefits patients can expect to derive from treatment. The ASCO-VF also takes the cost of a treatment into consideration, whereas the ESMO-MCBS focuses on the magnitude of benefit provided by a treatment. Cancer should not be treated, or viewed, empirically; however, empirical evidence of the true level of benefit derived by patients in clinical trials is still better than no evidence.

A pertinent example is provided by the move towards approval of agents on the basis of evidence from phase II trials, which typically have cohorts of fewer than 100 patients and usually noncomparative designs. This approach, in a period when so many new agents are becoming available for more-specifically defined populations of patients is, arguably, a pragmatic one, which has expedited the availability of both novel agents and those already approved for other indications, and is associated with considerably lower costs than traditional, large phase III trials. Others would argue, however, that the use of agents approved on the basis of data from trials that do not adequately compare such agents with standard-of-care treatments precludes the ability of patients and oncologists to make an evidence-based decision on the benefits versus adverse effects of novel agents in terms of comparative effect sizes. In their Comment article, regulatory experts from the FDA highlight that accelerated approvals based on single-arm trials are made with an agreement that data from comparative trials will soon be forthcoming. Furthermore, the FDA, and most likely other regulatory agencies, are moving towards the greater use of 'real-world' evidence, in addition to that obtained in the selected and nonrepresentative clinical trial cohorts. Use of such evidence would enable the indications of many agents to be expanded to the so-called rare cancers, and would likely incur fewer costs than small clinical trials for such indications.

All of these measures, as necessary and well intended as they are, fail to fully address the underlying issue of the rising and unsustainable costs of anticancer drugs. As highlighted in the Comment by Booth and Del Paggio, the CDK4/6 inhibitor palbociclib, which was approved in 2016 as a treatment of metastatic breast cancer and is available in the USA at a monthly cost of US\$13,155, was recently judged by the UK National Institute for Health and Care Excellence (NICE) as being too expensive for routine use in the UK National Health Service, despite a median improvement in progression-free survival of 10 months. As an independent public body, judgements made by NICE are arguably as good an indication as any of the true balance between the costs and benefits of new anticancer drugs, and palbociclib is certainly not the first anticancer drug to be deemed overly expensive for the benefits it provides, even though they seem substantial.

These various issues highlight one of the greatest challenges in clinical oncology - how best to pay for the treatments. Clearly, the drug-development process is expensive and risky, and the manufacturers of novel anticancer drugs do require sufficient funding to continue to innovate, but have we reached a point where this continued innovation becomes essentially futile owing to the prohibitive costs of treatments and our inability to appropriately test so many combinations in future trials? Will the pricing models employed by pharmaceutical companies and price negotiations in different healthcare systems change in order to facilitate the wider use of these products? Perhaps drug-approval decisions should be centred more on the extent of clinical benefit to the patient rather than on the statistical significance of trial outcomes. Treatment settings, previous lines of therapy, and prognosis should all be carefully considered in any scale. For instance, a 2-month survival benefit for heavily pretreated patients with terminal illness and a life expectancy below 3 months is not comparable to the same survival improvement for patients with a much longer life expectancy treated with curative intent. Regardless, future progress should be at a lower price! 